\title{
Decision Directed Channel Estimation Aided OFDM Employing Sample-Spaced and Fractionally-Spaced CIR Estimators
}

\author{
Jos Akhtman, Student Member, IEEE, and Lajos Hanzo, Fellow, IEEE
}

\begin{abstract}
In this letter we characterize the substantial difference between two channel estimation approaches, namely the sample-spaced (SS) and the fractionally-spaced (FS) channel impulse response (CIR) estimators. The achievable performance of decision-directed channel estimation (DDCE) methods employing both the SS- and the FS-CIR estimators is analyzed in the context of an OFDM system. The performance of the two estimation methods is compared and it is shown that the DDCE scheme employing the Projection Approximation Subspace Tracking (PAST)-aided FS-CIR estimator outperforms its SS-CIR estimator-based counterpart.
\end{abstract}

Index Terms-Multiuser OFDM, decision directed channel estimation, impulse response estimation SDMA.

\section{INTRODUCTION}

$\mathbf{T}$ HE family of well-documented decision directed channel estimation (DDCE) methods [1]-[4] is eminently suitable for OFDM-based systems [5], [6]. The major benefit of the DDCE scheme is that in contrast to purely pilot assisted channel estimation methods [7]-[10], both the pilot symbols as well as all the information symbols are utilised for channel estimation [1]. The simple philosophy of this method is that in the absence of transmission errors we can benefit from the availability of $100 \%$ pilot information by using the detected subcarrier symbols as an a posteriori reference signal. The employment of this method allows us to reduce the number of pilot symbols required.

Furthermore, a DDCE method suitable for employment in both OFDM and MC-CDMA systems was proposed in [11]. The estimator derived in [11] assumes a channel characterized by a Sample-Spaced Channel Impulse Response (SS-CIR). However, this assumption cannot be satisfied in realistic channel conditions, since we have no control over the delay of the CIR taps. Hence, the novel contribution of this letter is that we analyse the achievable performance of the SS-CIRbased DDCE scheme ${ }^{1}$ in conjunction with a more realistic Fractionally-Spaced (FS) CIR-based channel model. Furthermore, we propose an enhanced DDCE scheme invoking a Projection Approximation Subspace Tracking (PAST)-aided [12] FS-CIR estimator in contrast to the SS-CIR estimator.

Manuscript received April 26, 2005; revised May 20, 2006; accepted September 12, 2006. The associate editor coordinating the review of this letter and approving it for publication was C. Xiao. The work reported in this paper has formed part of the Wireless Enabling Techniques work area of the Core 3 Research Programme of the Virtual Centre of Excellence in Mobile and Personal Communications, Mobile VCE, www.mobilevce.com, whose funding support, including that of EPSRC, is gratefully acknowledged. Fully detailed technical reports on this research are available to Industrial Members of Mobile VCE.

The authors are with the School of ECS., Univ. of Southampton, SO17 1BJ, UK (e-mail: \{lh, j.akhtman $\} @$ soton.ac.uk)

Digital Object Identifier 10.1109/TWC.2007.05277.

${ }^{1}$ the channel estimation method considered was partly described in [11]
We then perform a comparison between the two methods considered and demonstrate the advantages of the proposed scheme.

The rest of this paper is structured as follows. Our channel model and the corresponding system model are described in Section II. The MMSE SS-CIR estimator introduced in [11] is briefly considered in Section III-B. The PAST-aided FSCIR estimator proposed is derived in Section III-C. Finally, the performance of both methods is compared using extensive computer simulations in Section IV, before concluding in Section V.

\section{SySTEM MODEL}

\section{A. Channel Statistics}

A Single Input Single Output (SISO) wireless communication link is constituted by a multiplicity of statistically independent components, termed as paths. The resultant channel is referred to as a multipath channel. Each individual path is considered to be a single distortionless ray between the transmitter and the receiver antennas.

The individual scattered and delayed signal components usually arise as a result of refraction or diffraction from scattering surfaces. In most of the recently proposed wireless mobile channel models each such CIR component $\alpha_{l}$ associated with an individual channel path is modelled by a wide sense stationary (WSS) narrow-band complex Gaussian process [13] having correlation properties characterised by the cross-correlation function

$$
r_{\alpha}[m, j]=E\left\{\alpha_{i}[n] \alpha_{j}^{*}[n-m]\right\}=r_{t ; i}[m] \delta[i-j],
$$

where $n$ is a discrete OFDM-symbol-related time-domain index and $\delta[\cdot]$ is the Kronecker delta function. The above equation suggests that the different CIR components are assumed to be mutually uncorrelated and each exhibits timedomain autocorrelation properties defined by the time-domain correlation function $r_{t ; i}[\mathrm{~m}]$. The Fourier transform pair of the correlation function $r_{t}[n]$ associated with each CIR tap corresponds to a band-limited power spectral density (PSD) $p_{t}(f)$, so that we have $p_{t}(f)=0$, if $|f|>f_{d}$, where $f_{d}$ is termed as the maximum Doppler frequency.

We adopt the complex baseband representation of the continuous-time Channel Impulse Response (CIR) given by [13]

$$
h(t, \tau)=\sum_{l} \alpha_{l}(t) c\left(\tau-\tau_{l}\right)
$$

where $\alpha_{l}(t)$ is the time-variant complex amplitude of the $l$ th path and $\tau_{l}$ is the corresponding path delay, while $c(\tau)$ is the aggregate impulse response of the transmitter-receiver 

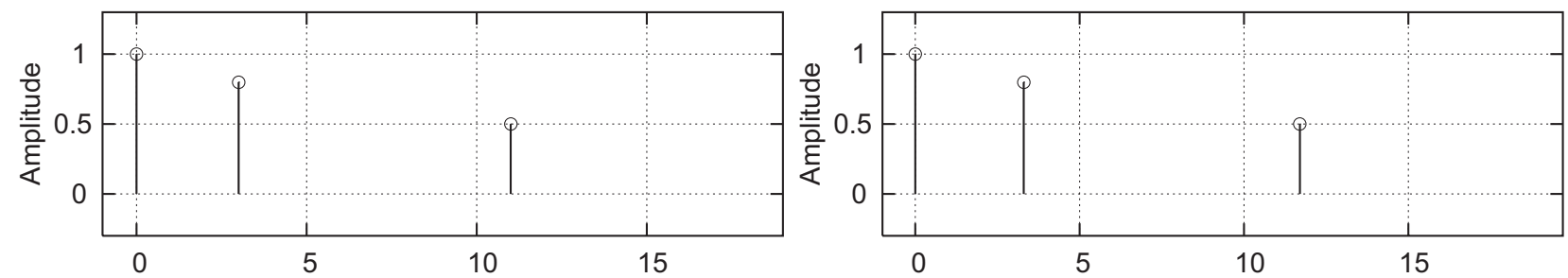

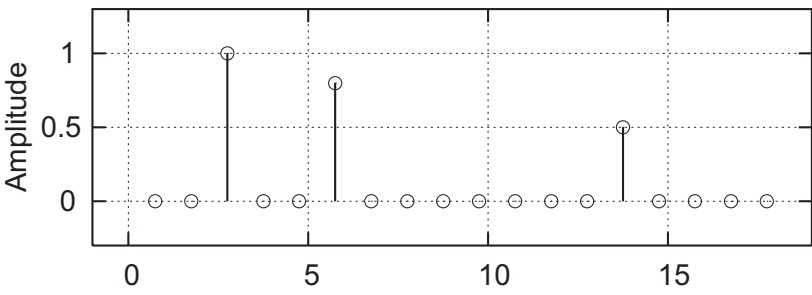

(a) Sample Index

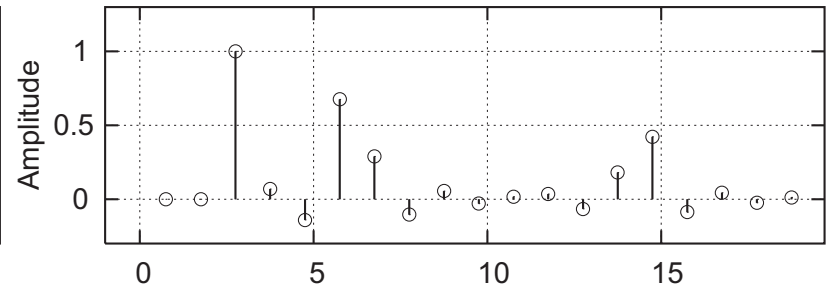

(b) Sample Index

Fig. 1. The FS-CIR (top) and the effective SS-CIR (bottom) resulting from the convolution of the original FS-CIR with the raised cosine filter impulse response for the cases of (a) sample-spaced and (b) fractionally-spaced power delay profiles.

pair, which usually corresponds to the raised-cosine Nyquist filter. From (2) the continuous channel transfer function can be expressed as

$$
\begin{aligned}
H(t, f) & =\int_{-\infty}^{\infty} h(t, \tau) e^{-\jmath 2 \pi f \tau} d \tau \\
& =C(f) \sum_{l} \alpha_{l}(t) e^{-\jmath 2 \pi f \tau_{l}},
\end{aligned}
$$

where $C(f)$ is the Fourier transform pair of the transceiver's impulse response $c(\tau)$.

As it was pointed out in [3], in OFDM/MC-CDMA systems using a sufficiently long cyclic prefix and adequate synchronisation [14], the discrete subcarrier-related Channel Transfer Function (CTF) can be expressed as

$$
\begin{aligned}
H[n, k]=H(n T, k \Delta f) & =C(k \Delta f) \sum_{l=1}^{L} \alpha_{l}[n] W_{K}^{k \tau_{l} / T_{s}} \\
& =\sum_{m=0}^{K_{0}-1} h[n, m] W_{K}^{k m},
\end{aligned}
$$

where

$$
h[n, m]=h\left(n T, m T_{s}\right)=\sum_{l=1}^{L} \alpha_{l}[n] c\left(m T_{s}-\tau_{l}\right)
$$

is the Sample-Spaced CIR (SS-CIR) and we have $W_{K}=\exp (-\jmath 2 \pi / K)$. The quantities $K, \quad L$ and $K_{0}$ denote the number of OFDM subcarriers, the number of fractionally-spaced channel paths and the number of equivalent sample-spaced CIR taps respectively, while $T_{s}$ is the base-band signal's sample duration. Note that in realistic channel conditions associated with non-sample-spaced timevariant path-delays $\tau_{l}(n)$ the receiver will encounter received signal components dispersed over several neighbouring samples owing to the convolution of the transmitted signal with the system's CIR, which we also refer to as leakage. This phenomenon is usually unavoidable and therefore the resultant SS-CIR $h[n, m]$ will be constituted of numerous correlated non-zero taps, as described by Equation (2) and illustrated in Figure 1. By contrast, the fractionally-spaced
CIR (FS-CIR) $\alpha_{l}[n]=\alpha_{l}(n T)$ will be constituted by a lower number of $L \ll K_{0} \ll K$ statistically independent non-zero taps associated with distinctive propagation paths, as depicted in Figure 1.

As it was shown in [3], the crosscorrelation function $r_{H}[m, l]$, which characterized both the time- and frequencydomain correlation properties of the discrete CTF coefficients $H[n, k]$ associated with different OFDM blocks and subcarriers can be described as

$$
\begin{aligned}
r_{H}[m, l] & =E\left\{H[n+m, k+l] H^{*}[n, k]\right\} \\
& =\sigma_{H}^{2} r_{t}[m] r_{f}[l],
\end{aligned}
$$

where $r_{t}[m]$ is the time-domain correlation function of Equation (1), while $r_{f}[i]$ is the frequency-domain correlation function, which can be expressed as in [8]

$$
r_{f}[l]=|C(l \Delta f)|^{2} \sum_{l=1}^{L} \frac{\sigma_{l}^{2}}{\sigma_{H}^{2}} e^{-j 2 \pi l \Delta f \tau_{i}},
$$

where $\sigma_{H}^{2}=\sum_{i=1}^{L} \sigma_{i}^{2}$. Consequently, the discrete frequencydomain model of the OFDM/MC-CDMA system can be described as

$$
y[n, k]=H[n, k] x[n, k]+w[n, k],
$$

for $k=0, \ldots, K-1$ and all $n$, where $y[n, k], x[n, k]$ and $w[n, k]$ are the received symbol, the transmitted symbol and the Gaussian noise sample respectively, corresponding to the $k$ th subcarrier of the $n$th OFDM block. Furthermore, $H[n, k]$ is the complex CTF coefficient associated with the $k$ th subcarrier and time instant $n$.

\section{Channel Estimation}

\section{A. Decision Directed Channel Estimator}

The schematic of the channel estimation method considered is depicted in Figure 2. Our channel estimator is constituted by what we refer to as an a posteriori decision-directed CTF estimator followed by a CIR estimator and an a priori CIR predictor [1]. As seen in Figure 2, the task of the CTF and CIR estimators is to estimate the CIR taps corresponding to 


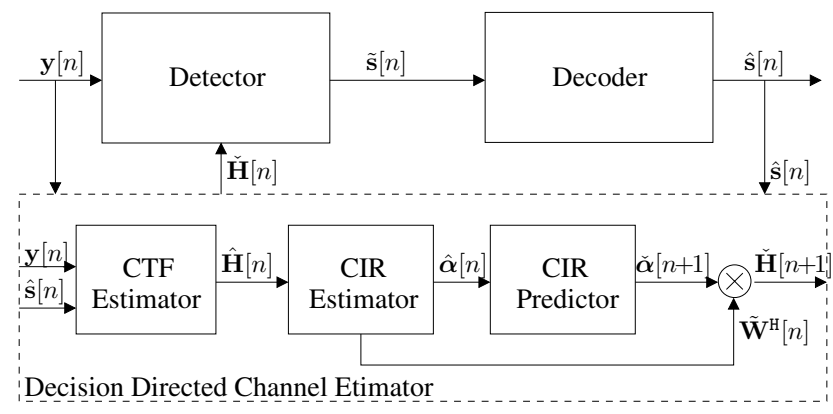

Fig. 2. Schematics of a generic receiver employing Decision Directed Channel Estimator constituted by an a posteriori decision-directed CTF Estimator, followed by a CIR Estimator and an a priori CIR predictor.

the current channel state based on the a posteriori information gleaned concerning the received subcarrier-related symbols.

More specifically, the a posteriori CTF estimator's inputs are the received subcarrier-related frequency-domain signal $\boldsymbol{y}[n]$ and the potentially error-infested decision-based estimate $\hat{\boldsymbol{s}}[n]$. The resultant CTF coefficients' estimate $\hat{\boldsymbol{H}}[n]$ is fed into the CIR estimator, which generates the estimate of the current sample-spaced or fractionally-spaced CIR, denoted as $\hat{\boldsymbol{h}}[n]$ or $\hat{\boldsymbol{\alpha}}[n]$, respectively. Subsequently, the estimated CIR taps are fed into the low-rank time-domain CIR tap predictor of Figure 2 for the sake of producing an a priori estimate $\check{\boldsymbol{\alpha}}[n+1]$ of the next CIR on a CIR tap-by-tap basis [1]. Finally, the predicted CIR is converted to the frequency-domain CTF with the aid of the tranformation matrix $\tilde{W}$. The resultant CTF is employed by the receiver for the sake of detecting and decoding the next OFDM symbol. Note that this principle requires the transmission of a pilot-based channel sounding sequence, such as for example in FD pilot-assisted OFDM, during the initialisation stage.

The CTF estimator of Figure 2 considered, constitutes an MMSE estimator [15], which may be characterized as follows. The MMSE estimator of the CTF coefficients $H[n, k]$ of the scalar linear model described by Equation (9), where the parameters $H[n, k]$ are assumed to be complex-Gaussian distributed with a zero mean and a variance of $\sigma_{H}^{2}$, is given by [15]:

$$
\begin{aligned}
\tilde{H}[n, k] & =\left(\frac{x^{*}[n, k] x[n, k]}{\sigma_{w}^{2}}+\frac{1}{\sigma_{H}^{2}}\right)^{-1} \\
& \cdot \frac{x^{*}[n, k] y[n, k]}{\sigma_{w}^{2}}=\frac{x^{*}[n, k] y[n, k]}{|x[n, k]|^{2}+\frac{\sigma_{w}^{2}}{\sigma_{H}^{2}}} .
\end{aligned}
$$

\section{B. MMSE SS-CIR Estimator}

The MMSE CTF estimates $\tilde{H}[n, k]$ of Equation (10) can now be modelled as complex Gaussian-distributed variables having a mean of $H[n, k]$, which represents the actual CTF coefficients encountered and a variance of $\sigma_{v}^{2}=M S E_{H}$, where $M S E_{H}$ is the average Mean Square Error (MSE) associated with the MMSE CTF estimator of Equation (10). Consequently, we have

$$
\tilde{H}[n, k]=H[n, k]+v[n, k],
$$

where $v[n, k]$ represents the i.i.d. complex-Gaussian noise samples having a zero mean and a variance of $\sigma_{v}^{2}$.
In the case of a sample-spaced CIR estimator we substitute the CTF of Equation (5) into (11), which yields

$$
\tilde{\boldsymbol{H}}[n]=\boldsymbol{W} \boldsymbol{h}[n]+\boldsymbol{v}[n] .
$$

The MMSE estimator of the SS-CIR taps $h[n, m]$ of the linear vector model described by Equation (12) is given by [15]

$$
\hat{\boldsymbol{h}}=\left(\boldsymbol{C}_{h}^{-1}+\boldsymbol{W}^{\mathrm{H}} \boldsymbol{C}_{v}^{-1} \boldsymbol{W}\right)^{-1} \boldsymbol{W}^{\mathrm{H}} \boldsymbol{C}_{v}^{-1} \tilde{\boldsymbol{H}},
$$

where we omit the time-domain OFDM-block-spaced index $n$ for the sake of notational simplicity and define $\boldsymbol{C}_{h}$ and $\boldsymbol{C}_{v}$ as the covariance matrices of the SS-CIR vector $\boldsymbol{h}$ and the scalar-MMSE CTF estimator's noise vector $\boldsymbol{v}$, respectively. The elements of the noise vector $\boldsymbol{v}$ are assumed to be complexGaussian i.i.d. samples and therefore we have $\boldsymbol{C}_{v}=\sigma_{v}^{2} \boldsymbol{I}$. On the other hand, as follows from the assumption of having uncorrelated SS-CIR taps, the SS-CIR taps' covariance matrix is a diagonal matrix $\boldsymbol{C}_{h}=\operatorname{diag}\left(\sigma_{l}^{2}\right)$, where $\sigma_{l}^{2}=$ $E\left\{|h[n, l]|^{2}\right\}$. Substituting $\boldsymbol{C}_{h}$ and $\boldsymbol{C}_{v}$ into Equation (13) yields

$$
\begin{aligned}
\hat{\boldsymbol{h}} & =\left(\operatorname{diag}\left(\frac{1}{\sigma_{l}^{2}}\right)+\frac{1}{\sigma_{v}^{2}} \boldsymbol{W}^{\mathrm{H}} \boldsymbol{W}\right)^{-1} \boldsymbol{W}^{\mathrm{H}} \frac{1}{\sigma_{v}^{2}} \tilde{\boldsymbol{H}} \\
& =\operatorname{diag}\left(\frac{\sigma_{l}^{2}}{\sigma_{v}^{2}+K \sigma_{l}^{2}}\right) \boldsymbol{W}^{\mathrm{H}} \tilde{\boldsymbol{H}},
\end{aligned}
$$

where we have exploited the fact that $\left[\boldsymbol{W}^{\mathrm{H}} \boldsymbol{W}\right]_{l, l^{\prime}}=$ $\sum_{k=0}^{K-1} e^{-\jmath 2 \pi \frac{k\left(l-l^{\prime}\right)}{K}}=K \delta\left[l-l^{\prime}\right]$ and therefore $\boldsymbol{W}^{\mathrm{H}} \boldsymbol{W}=K \boldsymbol{I}$, where $\boldsymbol{I}$ is a $\left(K_{0} \times K_{0}\right)$-dimensional identity matrix.

\section{FS-CIR Estimator}

By substituting the CTF of Equation (4) into (11) we arrive at

$$
\tilde{H}[n, k]=C(k \Delta f) \sum_{l=1}^{L} \alpha_{l}[n] W_{K}^{k \tau_{l} / T_{s}}+v[n, k],
$$

where, as previously, $C(f)$ is the frequency response of the transceiver's pulse-shaping filter, $W_{K}=e^{-\jmath 2 \pi \frac{1}{K}}$, while $\alpha_{l}[n]$ and $\tau_{l}$ are the amplitudes and the relative delays of the FSCIR taps, respectively. Equation (15) can be expressed in a matrix form as

$$
\tilde{\boldsymbol{H}}[n]=\tilde{\boldsymbol{W}} \boldsymbol{\alpha}[n]+\boldsymbol{v}[n],
$$

where we define the $(K \times L)$-dimensional matrix $\tilde{\boldsymbol{W}}=\operatorname{diag}(C[k]) \boldsymbol{W}$, in which $\operatorname{diag}(C[k])$ is a $(K \times K)$ dimensional diagonal matrix with the corresponding elements of the vector $C[k]$ on the main diagonal, while $W$ is the Fourier Transform matrix defined by $W_{k l}=W_{K}^{k \frac{\tau_{l}}{T_{s}}}$ for $k=-\frac{K}{2}, \cdots, \frac{K}{2}-1$ and $l=1, \cdots, L$.

It is plausible however, that in realistic wireless mobile channels, where at least one of the communicating terminals is in motion, the channel's PDP and the corresponding transformation matrix $\tilde{W}$ are time-variant and thus may not be known a priori at the receiver.

For the sake of accurate recursive tracking of the channel's Power Delay Profile we employ the so-called deflation Projection Approximation Subspace Tracking (dPAST) algorithm [12]. The deflation estimation strategy is based on the sequential Least Mean Square (LMS) estimation of the principal 


\begin{tabular}{cc}
\hline Algorithm 1 Deflation PAST & \\
\hline $\boldsymbol{H}_{1}[n]=\hat{\boldsymbol{H}}[n]$ & $(17 \mathrm{a})$ \\
for $\quad l=1,2, \ldots, L \quad$ do \\
$\quad \hat{\alpha}_{l}[n]=\boldsymbol{w}_{l}^{\mathrm{H}}[n-1] \boldsymbol{H}_{l}[n]$ \\
$\quad \lambda_{l}[n]=\eta \lambda_{l}[n-1]+\left|\hat{\alpha}_{l}[n]\right|^{2}$ \\
$\boldsymbol{e}_{l}[n]=\boldsymbol{H}_{l}[n]-\boldsymbol{w}_{l}[n-1] \hat{\alpha}_{l}[n]$ \\
$\boldsymbol{w}_{l}[n]=\boldsymbol{w}_{l}[n-1]+\boldsymbol{e}_{l}[n]\left(\hat{\alpha}_{l}^{*}[n] / \lambda_{l}[n]\right)$ \\
$\quad \boldsymbol{H}_{l+1}[n]=\boldsymbol{H}_{l}[n]-\boldsymbol{w}_{l}[n] \hat{\alpha}_{l}[n]$ \\
end for
\end{tabular}

components of the random random signal vector encountered. It may also be alternatively regarded as recursive tracking of the Singular Value Decomposition (SVD) of the time-variant channel's covariance matrix $\boldsymbol{R}_{H}[n]$. The method iteratively updates the eigenvalues and the corresponding eigenvectors of the channel's covariance matrix $\boldsymbol{R}_{H}[n]$. First we update the dominant eigenvalue $\alpha_{1}[n]$ and the corresponding eigenvector $\boldsymbol{w}_{1}[n]$ associated with the first row of the SVD transformation matrix $\tilde{\boldsymbol{W}}[n]$. Then the projection of the current observation vector $\boldsymbol{H}_{1}[n]$ onto the estimated eigenvector $\boldsymbol{w}_{1}[n]$ is subtracted from the observation vector itself, which results in retaining the second strongest eigenvalue as the dominant one. The process outlined above is then repeated, until the required estimation accuracy is accomplished.

The resultant PASTd-aided FS-CIR estimator is summarized in Algorithm 1, where in addition to the aforementioned quantities we employ the notations of the LMS forgeting factor $\eta$ as well as the $l$ th FS-CIR tap's running-average power $\lambda_{l}[n]$.

In order to complete the design of the DDCE scheme of Figure 2 we employ the a priori RLS CIR predictor derived in [16]. For the sake of brevity, we omit the derivation of the RLS predictor and refer to [16] for further details. The CIR predictor considered can be implemented in conjunction with both the SS-CIR and the FS-CIR estimators of Sections III-B and III-C, respectively.

\section{Simulation Results}

Our simulations were performed in the base-band frequency domain. We assume having a total bandwidth of $800 \mathrm{kHz}$. We considered an OFDM system, which utilised 128 QPSKmodulated orthogonal subcarriers. For forward error correction (FEC) we use $\frac{1}{2}$-rate turbo coding [17] employing two constraint-length $K=3$ Recursive Systematic Convolutional (RSC) component codes and a 124-bit WCDMA code interleaver [18]. The octally represented generator polynomials of $(7,5)$ were used. We employed the six-path Rayleigh-fading COST 207 Bad Urban (BU) channel model characterised in [19], having the maximum delay spread of $\tau_{\mathrm{a} m x}=6.6 \mu \mathrm{s}$ and different values of the OFDM-symbol-normalized Doppler frequency of $f_{D}$. In the DDCE scheme employed in the receiver we assumed having $K_{0}=16 \mathrm{SS}-\mathrm{CIR}$ taps and $L=6$ FS-CIR taps in the SS and FS modes, respectively.

Figure 3 demonstrates the channel estimation MSE exhibited by the DDCE scheme of Figure 2, employing both

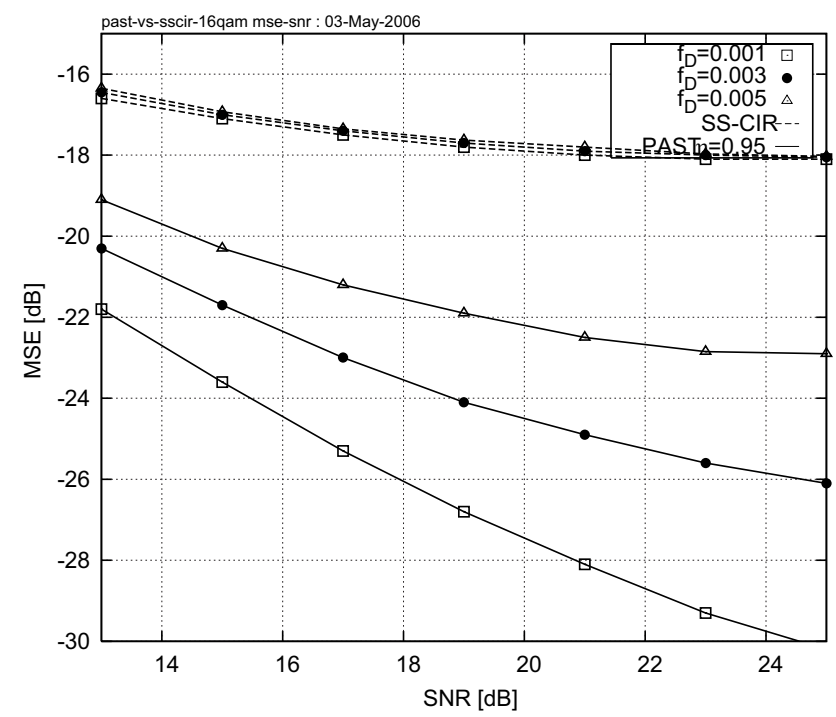

Fig. 3. Mean Square Error exhibited by the DDCE schemes employing SS-CIR and PAST-aided FS-CIR estimators of Sections III-B and III-C respectively, in the context of 16QAM-OFDM system.

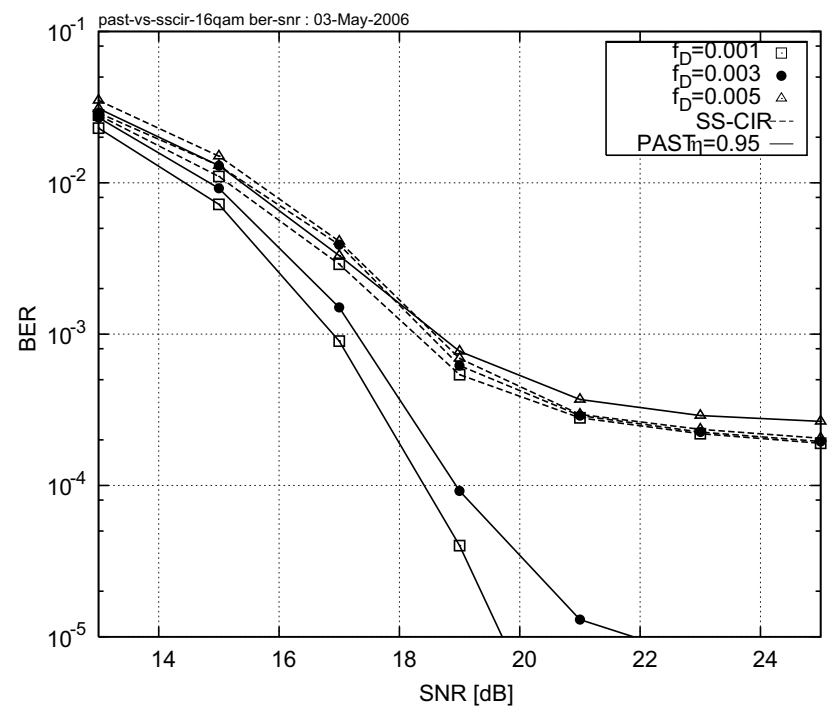

Fig. 4. Bit Error Rate performance exhibited by the 16QAM-OFDM system employing DDCE schemes based on SS-CIR as well as the PAST-aided FSCIR estimators of Sections III-B and III-C, respectively.

the SS-CIR estimator described in Section III-B and the FSCIR estimator derived in Section III-C. The corresponding achievable Bit Error Rate (BER) performance is depicted in Figure 4. The simulations were carried out over the period of 100,000 QPSK-modulated $K=128$-subcarrier OFDM/MCCDMA symbols. It can be seen in Figure 3 that the DDCE employing the a posteriori FS-CIR estimator-aided method outperforms its SS-CIR estimator-based counterpart over the entire range of the SNR values considered. Furthermore, the DDCE scheme utilizing the SS-CIR estimator exhibits an irreducible noise-floor at high SNR values. This effect can be explained by the fact that the SS-CIR-based estimator of Section III-B imposes a constraint of a circular symmetry on the FD-CTF $\hat{\boldsymbol{H}}[n]$. More specifically, the DFT transformation $\boldsymbol{W}$ of Equation 12 yields $\hat{H}[0, n]=\hat{H}[K, n]$, which evidently deviates from the actually encountered FS-CIR-based CTF 
having $H[0, n] \neq H[K, n]$, regardless of the encountered SNR.

Finally, the computational complexity of both the SS- and FS-CIR estimators is rather similar. More specifically, the total complexity imposed by the SS-CIR-based DDCE scheme using the FFT is of the order of $O\left(3 K+2 K \log _{2} K\right)$, where $K$ is the number of subcarriers and $O\left(K \log _{2} K\right)$ represents the complexity associated with the FFT operation. On the other hand, the complexity associated with the PAST-aided FS-CIR estimator may be approximated by $O(5 K L)$, where $L$ is the number of FS-CIR taps. The quantities $3 K+2 K \log _{2} K$ and $5 K L$ are of the same order, particularly in the case of having $K=128, L=6$, where we have $\log _{2} K=6$.

\section{COnClusions}

In this paper we characterized two DDCE schemes, namely the DDCE employing the a posteriori SS-CIR estimator derived in [11] and its PAST-aided FS-CIR estimator-based counterpart. The performance of both methods was explored in conjunction with realistic channel conditions characterized by a time-variant Rayleigh fading FS-CIR. We have shown that the latter estimation method exhibits substantial advantages in terms the achievable system performance. Our future research will provide channel estimators for a further range of cuttingedge OFDM systems, for example for the family of so-called direct Minimum Bit Error Ratio (MBER) OFDM modems [20] and for near-instantaneously adaptive OFDM [21].

\section{REFERENCES}

[1] L. Hanzo et al., OFDM and MC-CDMA for Broadband Multi-User Communications, WLANs and Broadcasting. relax John Wiley and IEEE Press, 2003, 992 pages.

[2] O. Edfords et al., "OFDM channel estimation by singular value decomposition," IEEE Transactions on Communications, vol. 46, pp. 931-939, July 1998.

[3] Y. Li, L. Cimini, and N. Sollenberger, "Robust channel estimation for OFDM systems with rapid dispersive fading channels," IEEE Trans. Commun., vol. 46, no. 7, pp. 902-915, Apr. 1998.

[4] M. Munster and L. Hanzo, "Parallel interference cancellation assisted decision-directed channel estimation for ofdm systems using multiple transmit antennas," IEEE Trans. Wireless Commun., vol. 4, no. 5, pp. 2148-2162, Sept. 2005.
[5] T. Keller and L. Hanzo, "Adaptive multicarrier modulation: a convenient framework for time-frequency processing in wireless communications," in Proc. IEEE, vol. 88, no. 5, pp. 611-640, May 2000.

[6] - "Adaptive modulation techniques for duplex OFDM transmission," IEEE Trans. Vehic. Technol., vol. 49, no. 5, pp. 1893-1906, Sept. 2000.

[7] P. Höher, S. Kaiser, and P. Robertson, "Pilot-symbol-aided channel estimation in time and frequency," in IEEE Global Telecommunications Conference: The Mini-Conference, Phoenix, AZ, Nov. 1997, pp. 90-96.

[8] Y. Li, "Pilot-symbol-aided channel estimation for OFDM in wireless systems," IEEE Trans. Vehic. Technol., vol. 49, pp. 1207-1215, July 2000.

[9] F. Sanzi and J. Speidel, "An adaptive two-dimensional channel estimation for wireless OFDM with application to mobile DVB-T," IEEE Trans. Broadcasting, vol. 46, no. 2, pp. 128-133, June 2000.

[10] M.-X. Chang and Y. Su, "Model-based channel estimation for OFDM signals in Rayleigh fading," IEEE Trans. Commun., vol. 50, pp. 540544, Apr. 2002.

[11] J. Akhtman and L. Hanzo, "Generic reduced-complexity MMSE channel estimation for OFDM and MC-CDMA," in Proc. Spring'05 IEEE Vehic. Technol. Conf., Stockholm, Sweden, May 30 - June 12005.

[12] B. Yang, "Projection approximation subspace tracking," IEEE Trans. Signal Processing, vol. 43, no. 1, pp. 95-107, January 1995.

[13] R. Steele and L. Hanzo, Eds., Mobile Radio Communications, 2nd ed. New York, USA: John Wiley and IEEE Press, 1999, 1090 pages.

[14] T. Keller et al., "Orthogonal frequency division multiplex synchronization techniques for frequency-selective fading channels," IEEE J. Select. Areas Commun., vol. 19, no. 6, pp. 999-1008, June 2001.

[15] S. M. Kay, Fundamentals of Statistical Signal Processing. Englewood Cliffs, NJ, USA: Prentice-Hall, 1998.

[16] D. Schafhuber and G. Matz, "MMSE and adaptive prediction of timevarying channels for OFDM systems," IEEE Trans. Wireless Commun., vol. 4, no. 2, pp. 593-602, Mar. 2005.

[17] L. Hanzo, T. H. Liew, and B. L. Yeap, Turbo Coding, Turbo Equalisation and Space-Time Coding. Chichester, UK; Piscataway, NJ, USA: John Wiley and IEEE Press, 2002, 766 pages. (For detailed contents, please refer to http://www-mobile.ecs.soton.ac.uk.).

[18] H. Holma and A. Toskala, Eds., WCDMA for UMTS : Radio Access for Third Generation Mobile Communications. John Wiley and Sons, Ltd., 2000.

[19] M. Failli, "Digital land mobile radio communications COST 207," European Commission, Tech. Rep., 1989.

[20] M. Y. Alias, S. Chen, and L. Hanzo, "Multiple-antenna-aided OFDM employing genetic-algorithm-assisted minimum bit error rate multiuser detection," IEEE Trans. Vehic. Technol., vol. 54, no. 5, pp. 1713-1721, Sept. 2005.

[21] B. J. Choi and L. Hanzo, "Optimum mode-switching-assisted constantpower single- and multicarrier adaptive modulation," IEEE Trans. Vehic. Technol., vol. 52, no. 3, pp. 536-560, May 2003. 